\title{
Age at death and rectangularisation of the survival curve: trends in Switzerland, 1969-1994
}

\author{
Fred Paccaud, Claudio Sidoti Pinto, Alfio Marazzi, Judith Mili
}

\begin{abstract}
Objective-To check if signs of rectangularisation of the survival curve appeared during recent decades in Switzerlandthat is, if life expectancy is approaching a maximum with a clustering of age at death around an average value (the so called "compression of mortality").

Methods-Descriptive analysis of age of death and its trends over 26 years, as characterised by the modal value, median, and various percentiles beyond the median.

Population-All deaths occurring after the 50th birthday in Switzerland between 1969 and 1994 ( $n=1390$ 362).

Main results-Age at death is increasing at a sustained rate at all percentiles equal or greater than 50 , without any slow down in the trend during this period. The increase is more marked among women. Rates of increase are diminishing as the percentiles of age at death are higher, suggesting some clustering of deaths beyond the median value. However, the maximum age at death, if any, seems to be far from the current
\end{abstract}

median values, even for women who enjoy a relatively high median age at death.

(F Epidemiol Community Health 1998;52:412-415)

In 1980, Fries ${ }^{1}$ presented a new development of an old theory-that is, the "rectangularisation" of the survival curve. Among other aspects, this theory states that the current decrease in mortality among old people is limited by a maximum life span. ${ }^{2}$ This should correspond to a delayed and sharp increase in mortality around some optimal value of duration of life in the human species.

The purpose of this paper is to analyse the recent trend in the distribution of age at death among old people in Switzerland, with a specific effort to identify any pattern compatible with a rectangularisation of survival.

\section{Population and methods}

Death certificates were obtained from the Federal Office of Statistics in Bern. The original set contains all deaths of Swiss residents during the period 1969-94 ( $\mathrm{n}=1537989$ deaths). The records contain complete dates of birth,
Institute for Social and Preventive Medicine, School of Medicine, University of Lausanne, Switzerland

Correspondence to: Professor F Paccaud, IUMSP, Bugnon 17, 1005 Lausanne, Switzerland.

Accepted for publication 16 September 1997
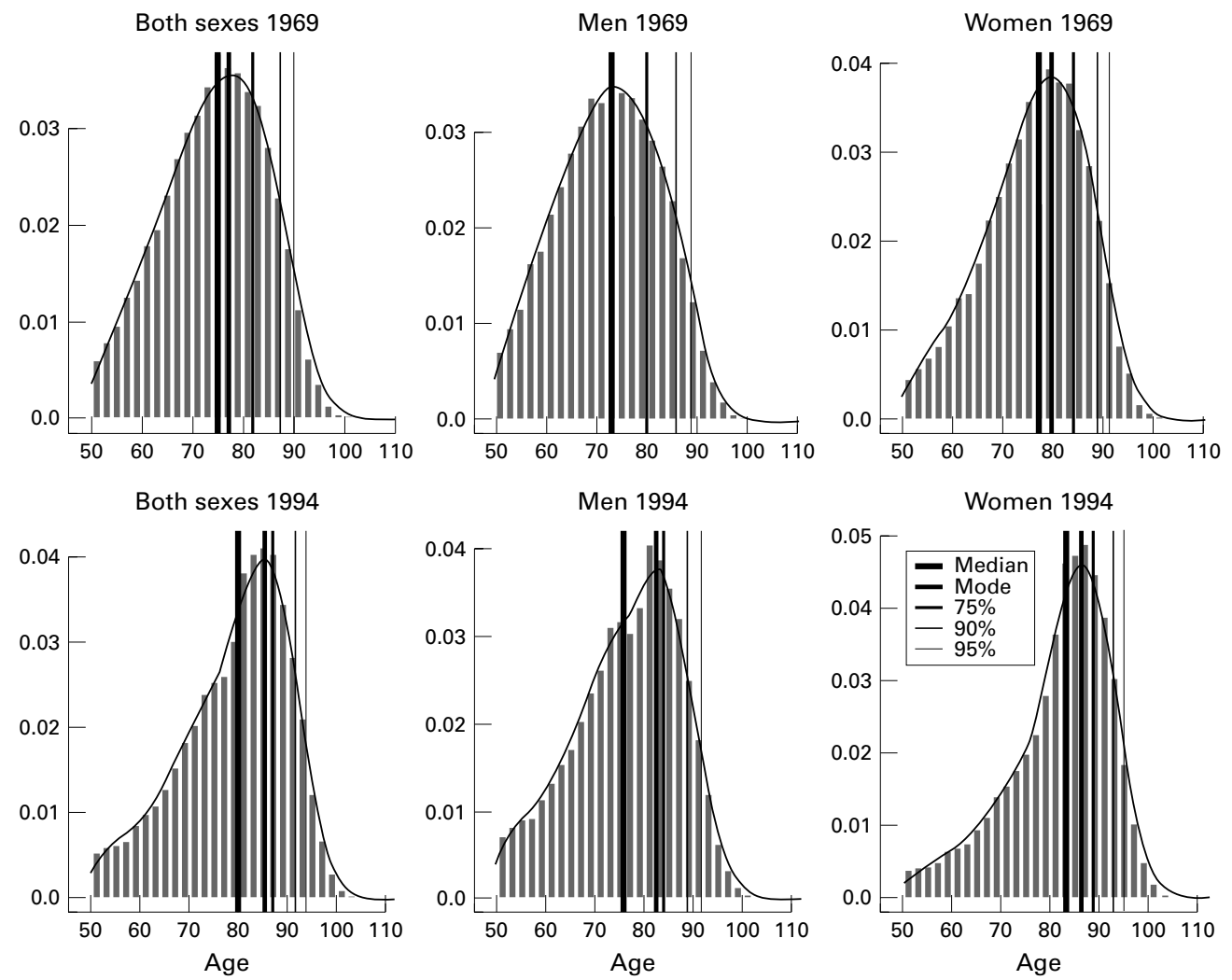

Figure 1 Histograms of age at death, Switzerland, 1969 and 1994. 
Table 1 Selected indicators of age at death, Switzerland, 1969 and 1994

\begin{tabular}{|c|c|c|c|c|c|c|c|}
\hline & & & Mode & Median & $\begin{array}{l}75 \% \text { (difference } \\
\text { with the median) }\end{array}$ & $\begin{array}{l}90 \% \text { (difference } \\
\text { with the median) }\end{array}$ & $\begin{array}{l}95 \% \text { (difference } \\
\text { with the median) }\end{array}$ \\
\hline 1 & Men & 1969 & 73 & 73 & $80(7)$ & $86(13)$ & $89(16)$ \\
\hline 2 & & 1994 & 82 & 78 & $85(7)$ & $89(11)$ & $92(14)$ \\
\hline 3 & & difference $1994-1969$ & 9 & 5 & 5 & 3 & 3 \\
\hline 4 & Women & 1969 & 79 & 77 & $84(7)$ & $88(11)$ & $91(14)$ \\
\hline 5 & & 1994 & 86 & 84 & $89(5)$ & $93(9)$ & $95(11)$ \\
\hline 6 & & difference 1994-1969 & 7 & 7 & 5 & 5 & 4 \\
\hline
\end{tabular}
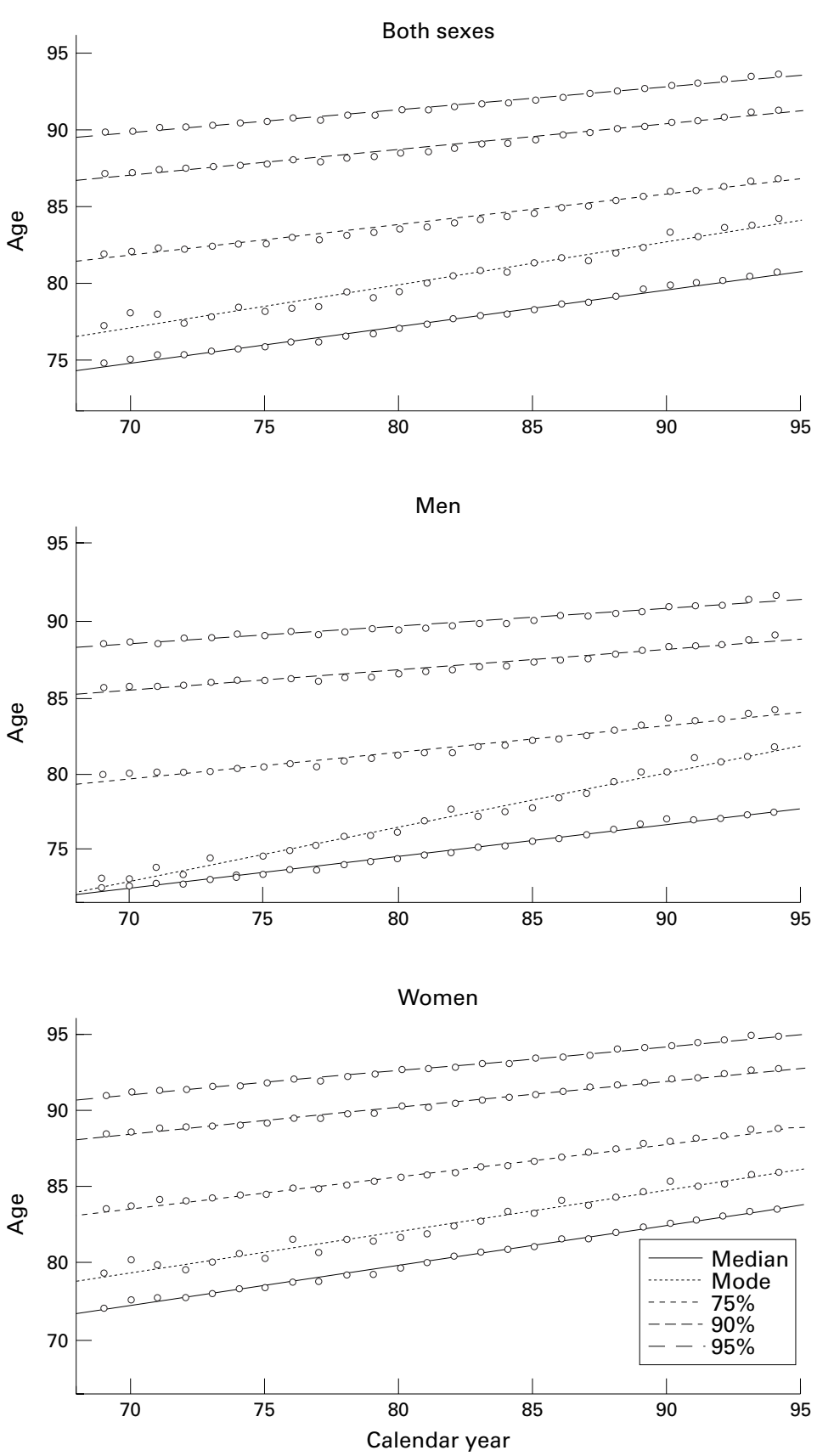

Figure 2 Trends of mode and percentiles of age at death, Switzerland, 1969-1994.

Table 2 Indicators of trends of age at death, Switzerland, 1969-1994

\begin{tabular}{|c|c|c|c|c|c|c|}
\hline & & Mode & Median & $75 \%$ & $90 \%$ & $95 \%$ \\
\hline \multirow[t]{2}{*}{ Men } & constant & 47.54 & 57.22 & 66.75 & 75.45 & 79.64 \\
\hline & slope & 0.37 & 0.22 & 0.19 & 0.14 & 0.13 \\
\hline \multirow[t]{2}{*}{ Women } & constant & 59.88 & 58.28 & 67.80 & 75.24 & 79.03 \\
\hline & slope & 0.28 & 0.27 & 0.23 & 0.19 & 0.17 \\
\hline
\end{tabular}

death, and sex. Only deaths occurring after the 50th birthday $(n=1390362$-that is, $90 \%$ of all deaths) were considered in this paper.

For each calendar year, the distribution percentiles of $50 \%, 75 \%, 90 \%$, and $95 \%$ of age at death were computed. Moreover, the modal age was estimated as the maximum of a non-parametric density estimate. ${ }^{3}$ All computations were made with age in days; however, results are presented in years (the number of days was divided by 365 and rounded to the nearest integer).

\section{Results}

Figure 1 shows the distribution of age at death at the beginning (1969) and at the end (1994) of the period under study. The increase of the mode and the median is obvious in both sexes: the shift of the median amounts to five years for men and seven years for women (table 1). The higher percentiles $(75 \%, 90 \%$ and $95 \%)$ also increase. The shift of the mode is more important for men (nine years) than for women (seven years).

Figure 1 suggests that the right hand sides of the 1994 histograms of the distributions are steeper than those of the 1969 histograms. Quantitative estimates of these differences are given in table 1: the gap between the median and the higher percentiles decreases during the period under study, for both sexes. For example, the difference between the median and the 95th percentile amounts to 16 years for men and 14 years for women in 1969, but it is only 14 and 11 years in 1994, respectively. Trends in differences are similar for the other percentiles for both sexes. In both years 1969 and 1994, the gap between the median and the higher percentiles is shorter for women than for men. Moreover, the male percentiles of year 1994 (table 1, line 2) are close to the female percentiles of year 1969 (table 1, line 4): in fact, the pattern of age at death for women anticipated the male pattern by 20 or 30 years.

Figure 2 displays the trends of the modal values and of percentiles $(50 \%, 75 \%, 90 \%$, and 95\%) of age at death between 1969 and 1994 . For all percentiles, there is a regular increase during the period, with a striking linear trend (that is, the variability around the straight line is minimal).

Examination of figure 2 suggests that the straight lines converge. This confirms the impression from figure 1 that the distance between the percentiles beyond the median is shrinking over the period. This convergence is quantified in table 2 with the line coefficients (constants and slopes): the slopes are decreasing with the increasing percentiles beyond the median. 


\section{Discussion}

This study analyses the secular trends of age at death over 26 years in Switzerland, with a focus on people dying after the age of 50-that is, on the population most likely to die from "natural death". There is a linear increase in age at death: for the median, the rate of increase lies between 0.2 (men) and 0.3 (women) years of life per calendar year. There are no signs of a slowing down in either sex. An increase in age at death is also observed for people dying after the median age, although the annual rate of increase diminishes with increase in age. This latter finding suggests some reduction of the variability of age at death - that is, a compression of mortality.

Age at death is not an ideal measure of trends in mortality: any shift might be caused by a change of either the mortality or the cohort size. In that perspective, the most appropriate method is the construction of extinct cohorts life tables. However, age at death has definite advantages as an empirical measure of mortality $^{4}$ and has been used by several authors..$^{5-7}$ Among others, an important advantage of age at death is to overcome the problems related to the quality of data: distribution of age at death relies on a single source (death certificate), while the death rate is based on two sources of data (death certificates and census). As the quality of census data may be questionable among old people (both in terms of enumeration and age certification), it is an advantage to work on the "numerator" only (the age at death). In practice, age at death is appropriate to assess short-term trends as far as the change in cohort size is limited. ${ }^{5}$ These changes were negligible in Switzerland during the period under study, as shown in a separate analysis: thus, mortality effects dominate at late ages in shaping the distribution of age at death.

Findings gained in this study do not provide straightforward arguments in favour or against the rectangularisation of survival in Switzerland. On the one hand, several results are compatible with Fries' theory. There is a sustained increase in age at death, with a shift in distribution toward the right hand side (fig 1 and fig 2, table 2). Furthermore, the rate of secular increase diminishes with increasing percentiles (table 2), suggesting a reduction in the variability of age at death. The gender difference provides further arguments in the same direction: compared with men, women enjoy a higher age at death, and the distribution after the median is steeper for women than for men.

On the other hand, several results do not fit with any ongoing rectangularisation. For this 26 year period, the striking linearity of the increase in age at death does not slow down, either for the median or for the higher percentiles (fig 2). A separate analysis shows that, during the same period, the number of Swiss people surviving their 90th birthday increased by $32 \%$, and their 100 th birthday by $19 \%$. Furthermore, the rates of increase (the slopes in table 2) are higher for women than for men, at each percentile: in fact, one would expect a

\section{KEY POINTS}

- Improvement of the health status of the elderly and rectangularisation of the survival curve are still debated as hypothesis.

- Rectangularisation of the survival curve refers to an increasing mean age at death with a decreasing variability around the mean.

- Switzerland already enjoys an increased median age at death, which is however still increasing in both sexes.

- There is some convergence of the slopes of age at death in the higher percentiles, suggesting early signs of compression.

lower rate of increase among women, because women have already reached a higher age at death.

Similar studies have been conducted in various countries, showing conflicting results. A Dutch study ${ }^{8}$ presents data on mortality between 1952 and 1992. Overall, the results are in favour of rectangularisation of the survival curve, with an increase in life expectancy and a decline in variability of age at death. These signs of rectangularisation are less marked for people dying after the age of 60 than for younger adults, but are nevertheless observable.

In contrast, several studies from the United States provide arguments against rectangularisation. One study ${ }^{5}$ on the period 1962-1979 shows an increase in both the mean age at death and its variability (as measured by the standard deviation). Moreover, both the variability and the increase in variability are higher for women than for men despite the fact that women enjoy a higher mean age at death and a faster rate of increase (that is, an observation similar to the one made in this study). Another American study on mortality in old age between 1960 and $1990^{6}$ confirms the lack of compression of mortality. Similar arguments against any compression of mortality are reported from analyses made in California, ${ }^{9}$ in Australia, ${ }^{4}$ and in France. ${ }^{10}$ One study ${ }^{7}$ based on the trend in age at death between 1962 and 1984 in the US suggests a sort of "partial compression of mortality": there is a shift of deaths into the upper ages, but with an increasing variability of age at death.

Taken as a whole, the results from this study and from the literature suggest that the maximal mean age at death is certainly higher than 85 years, which is the value originally proposed by Fries. ${ }^{1}$ If any, a rectangularisation should thus occur at a higher age. Considering the excellent linearity shown in figure 2, the convergence of the trends can be used to estimate the age (and the calendar year, not shown here) at which the various pairs of lines will cross over (that is, at an age with a high concentration of deaths). Using the coefficients from table 2, the crossover of the various lines will occur at an age at death varying between 100 and 134 years for men, between 111 and 123 years for women. Predictions for women 
are less scattered and might be more credible because women already enjoy a longer life expectancy in Switzerland. These predictions grossly correspond to several other estimates. $^{6891112}$ For the moment, the well documented maximum life span is a 122 year old French woman.

If the upper limit of age at death is as high as 120 years (that is, still far from the current mean age at death), this is compatible with the sustained rate of increase at all ages in Switzerland. The slight convergence of the trends for the percentiles might be an early sign of compression of mortality. As noted before, these signs may be more visible in Switzerland (or in the Netherlands) than in the US, because these two European countries are in a more advanced stage of mortality transition. In fact, the median age at death is lower in the US than in Switzerland: this might explain that an expansion of mortality (rather than a compression) is still dominant in the US, while a compression of mortality is emerging in the two European countries.

At each percentile, the rate of increase in age at death is higher among women than men. This pattern is found in most studies, including those from the United States, ${ }^{6}$ and seems independent of the calendar period. This might be related to a gender specific difference in the upper limit of the life span.

\section{Conclusion}

The scientific theory of a fixed duration of life ${ }^{11}$ is not new and was first expressed by Buffon two centuries ago (quoted by Robine ${ }^{10}$ ). The development of genetics now suggests the mechanisms underlying a fixed life span, at least in animal models. ${ }^{13}{ }^{14}$ However, convincing tests to assess rectangularisation are difficult to develop and apply to empirical data. ${ }^{815}$ One difficulty is that any upper limit of life span is unlikely to be homogenous, even if environmental heterogeneity is reduced among individuals ${ }^{16}$ : some variation around the maximum life span will still be observable, related either to a genetic mechanism and selection of "long living" genes ${ }^{17}{ }^{18}$ or to residual factors like those related to social classes ${ }^{19}$ or ethnicity. ${ }^{9}$ Thus, the question is to estimate the amount of variation around this mean age at death.

Moreover, rectangularisation should not be seen as an output of mortality analysis, ${ }^{16}$ but as a part of an underlying process related to an improvement in the health status of the elderly ${ }^{1}$ : the period of illness and disablement is expected to be limited around the age at death, against authors predicting a pandemia of chronic diseases. ${ }^{20}$ There are several direct $\mathrm{t}^{102122}$ and indirect ${ }^{23}$ evidences that health status is currently improving among the elderly, with a corresponding increase in the so called "disability-free life expectancy".

It is in this perspective that the monitoring of age at death in older people should be made. Future studies should compare the evolution of age at death in various countries at different stages of mortality transition at advanced age, using similar measures of mortality. This will help for a better descriptive epidemiology of age at death and a better understanding of the mechanism of senescence. ${ }^{12}{ }^{24}$ The monitoring of age at death is also relevant for health services research and management, for a better estimate of the number of old people in the future, ${ }^{1125}$ and for a better prevision of the needs of aged people. ${ }^{24} 26$

Funding: Institute for Social and Preventive medicine, School of Medicine, University of Lausanne.

Conflicts of interests: none.

1 Fries JF. Aging, natural death and the compression of the morbidity. N Engl f Med 1980;303:130-5.

2 Schächter F, Cohen D, Kirkwood T. Prospects for the genetics of human longevity. Hum Genet 1993;91:519-26. ZS-Plus, advanced data analysis software. Seattle, WA: ZS-Plus, advanced data analysis
Statsci Division of Mathsoft, 1995.

4 Myers GC. Recent trends in mortality among the aged and their implications. In: McDonald JM, Everitt AV, Wheeler PJ, eds. Aging in Australia. Sydney: Australian Association of Gerontology, 1979.

5 Myers GC, Manton KG. Compression of mortality: myth or reality (I) ? Gerontologist 1984;24:346-53.

6 Manton KG, Stallard E. Longevity in the United States: Age and sex-specific evidence on life span limits from mortality patterns 1960-1990. F Gerontol A Biol Sci Med Sci 1996;51A:B362-375.

7 Rothenberg R, Lentzner HR, Parker RA. Population aging patterns: the expansion of mortality. I Gerontol B Psychol Sci Soc Sci 1991;46: S66-70.

8 Nusselder WJ, Mackenbach JP. The rectangularization of the survival curve in Netherlands, 1950-1992. Gerontologist 1996;36:773-82.

9 Go CC, Brustrom JE, Lynch MF, Aldwin CM. Ethnic trends in survival curves and mortality. Gerontologist 1995; 35:318-26.

10 Robine JM, Mormiche P, Cambois E. Evolution des courbes de survie totale, sans maladie chronique et sans incapacité en France de 1981 à 1991: application d'un modèle de 'OMS. Annales de démographie historique (Paris) 1996:99115 .

11 Olshansky SJ, Bruce CA, Cassel C. In search of Methuselah: Estimating the upper limits to human longevity. Science 1990;250:634-40.

12 Olshansky JS. Simultaneous/Multiple cause-delay (SIMCAD): An epidemiological approach to projecting mortality. F Gerontol 1987;42:358-65.

13 Carey JR, Liedo P, Orzoco D, et al. Slowing of mortality rates in older in large medfly cohorts. Science 1992;258: 457-60.

14 Brook A, Lithgow G, Johnson T. Mortality rates in a genetically heterogenous population of Caenorhabditis elegans. Science 1994;263:668-71.

15 Manton KG, Tolley HD. Rectangularization of the survival curve: implications of an ill posed question. 7 Aging Health curve: implications

16 Manton KG, Stallard E, Tolley HD. Limits to human life expectancy: evidence, prospects, and implications. Population and Development Review 1991;17:603-37.

17 Osamu S, Osimura M, Koi M, et al. Induction of cellular senescence in immortalized cell by human chromosome. Science 1990;247:707-10.

18 Kenyon C, Chang J, Gensch E, et al. A C Elegans mutant that lives twice as long as wild type. Nature 1993;366:461-

19 Desplanques G. L'inégalité devant la mort. Données Sociales 1993;5:251-8.

20 Grueneberg EM. The failure of success. Milbank $Q$ 1977;55:3-24.

21 Lentzner HR, Pamuk ER, Rhodenhiser EP, et al. The quality of life in the year before death. Am f Public Health 1992; 82:1093-8.

22 Nusselder WJ, Van der Velden K, Van Sonsbeek JLA, et al. The elimination of selected chronic diseases in a population: the compression and expansion of morbidity. Am $f$ Public Health 1996;86:187-94.

23 Lubitz J, Beebe J, Baker C. Longevity and Medicare expenditures. N Engl F Med 1995;332:999-1003.

24 Christensen K, Vaupel JW. Determinants of longevity: genetic, environmental and medical factors. F Int Med 1996;240:333-41.

25 Vaupel JW, Gowan AE. Passage to Methuselah: Some demographic consequences of continued progress against mortality. Am F Public Health 1986;76:430-3.

26 Watkin DM. Goal: " Rectangularize" survival; objective: change behavior. Clin Geriatr Med 1987;3:237-52. 